\section{BioMarin nabs first paediatric priority review voucher}

Regulators reward company for developing an enzyme replacement therapy for a rare paediatric indication with only 800 estimated patients in the United States.

The lowdown: In 2012, the FDA Safety and Innovation Act (FDASIA) introduced a new priority review voucher (PRV) to encourage drug makers to develop therapies for rare paediatric diseases. Modelled after the tropical disease priority review voucher (TD-PRV), the paediatric PRV entitles a successful sponsor of a rare paediatric disease drug to an 8-month priority review of the drug of its choice.

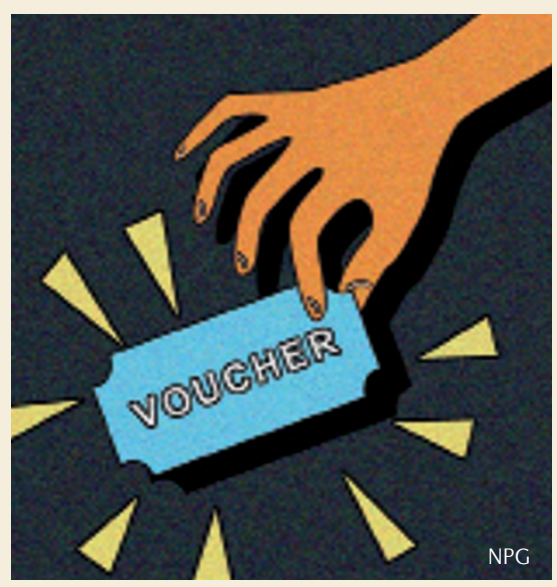

With the US Food and Drug Administration (FDA)'s approval of the enzyme replacement therapy elosulfase alfa for patients with mucopolysaccharidosis type IVA (also known as Morquio A syndrome), BioMarin became the first company to receive a paediatric PRV. BioMarin tested its drug in patients aged $5-57$ years old.

PRVs, and the 4 months faster market access they enable, have been estimated to be worth between US\$50 million and \$300 million. To date, only two TD-PVRs have been granted, to Novartis for Coartem (artemether plus lumefantrine) and to Janssen for bedaquiline. Novartis used its PRV to expedite the review of canakinumab in gouty arthritis, but the FDA rejected the application (Nature Rev. Drug Discov. 10, 721; 2011). As Nature Reviews Drug Discovery went to press, a regulatory decision was pending on the approval of Paladin's hexadecylphosphocholine, for leishmaniasis, which is eligible for a third TD-PRV. But whereas a TD-PRV can only be sold once to a new sponsor, the paediatric PRV can be traded multiple times, potentially increasing its value and the likelihood of a sale.

A limited, but unset, number of paediatric PRVs are up for grabs: the FDA will stop granting them after the last day of a 1-year period that will begin when the third voucher is awarded.

\section{FDA releases first chronic fatigue guidance}

Patient-reported outcomes are key to approval in this challenging indication, say regulators.

The lowdown: Between 1 million and 4 million people in the United States have chronic fatigue syndrome/myalgic encephalomyelitis (CFS/ME), but there are no approved therapies for this indication. To help bridge this gap, the US Food and Drug Administration (FDA) published a first CFS draft guidance, with guidelines around various aspects of trial designs.

Efficacy end points "should include patient-reported symptoms using well-defined and reliable [patient-reported outcome] instruments", says the agency. It lists CFS symptoms as including fatigue, unrefreshing sleep, weakness, muscle and joint pain, impaired memory or mental concentration, tender lymph nodes, sore throat, headaches and sleep dysfunction. "The FDA is not aware of existing [patient-reported outcome] instruments or sets of instruments optimal for measurement of fatigue or other symptoms of CFS/ME," it adds, but will consider proposals. Increased exercise capacity and reduced post-exertional malaise are also included as possible treatment benefits that may be measurable through either patient-reported outcomes or objective outcomes. The agency says it will consider the use of instruments that measure health-related quality of life as well.

The agency recommends that sponsors carry out two 24-week placebo-controlled trials to generate approvable, pivotal efficacy data. Because patients are likely to use treatments over prolonged periods, the FDA advises sponsors to collect long-term safety data, including, when efficacy trials are substantially less than 1 year, separate long-term safety trials.

The draft guidance document stemmed from the FDA's patient-focused drug development programme, through which the FDA is meeting with patient communities to get input on drug development priorities (Nature Rev. Drug Discov. 12, 651-652: 2013). CFS patients and their advocates were the first community to meet with the FDA, in April 2013. The agency is due to hold a total of 20 meetings with patients from different disease areas by the end of 2017.

\section{Regulators defend incretin drugs}

GLP1 mimetics and DPP4 inhibitors do not seem to be associated with pancreatic safety events, say US and European regulators in a joint paper. The lowdown: Last year, US and European drug regulators initiated safety reviews of two approved classes of incretin-based drugs: glucagon-like peptide 1 (GLP1) mimetics and dipeptidyl peptidase 4 (DPP4) inhibitors, which block the proteolytic degradation of incretins such as GLP1. At issue was whether these widely used diabetes drugs increased the risk of pancreatitis and pancreatic cancer, as suggested by biopsy samples of organ donors with type 2 diabetes who had been treated with incretin-based drugs or with other drugs (Diabetes 62, 2595-2604; 2013).

One year on, following a thorough review of animal and human data, the US Food and Drug Administration (FDA) and the European Medicines Agency are standing behind the safety profiles of the drugs (N. Engl.J. Med. 370, 794-797; 2014). "Both agencies agree that assertions concerning a causal association between incretin-based drugs and pancreatitis or pancreatic cancer, as expressed recently in the scientific literature and in the media, are inconsistent with the current data," they write. The agencies have not, however, reached a final conclusion, they add, and will continue to monitor the situation. "Although the totality of the data that have been reviewed provides reassurance, pancreatitis will continue to be considered a risk associated with these drugs until more data are available," they write.

The regulators point to various data to back their view of the safety profiles of these drugs. A re-evaluation of over 250 toxicology studies in 18,000 healthy animals, as well as new toxicity studies in rodent models of diabetes carried out by the FDA and by drug sponsors, didn't find any fresh red flags. On the human side, a review of clinical safety databases found small imbalances in the incidence of pancreatitis with incretin-based therapies in pre-marketing trials, but "the overall number of events was small". A meta-analysis of the DPP4 inhibitor sitagliptin and two long-term cardiovascular outcomes trials of the DPP4 inhibitors saxagliptin and alogliptin did not find compelling differences either, the agencies write. 